\title{
Sistema compatible de ahusamiento-volumen comercial para Swietenia macrophylla King (caoba) en Quintana Roo, México
}

\section{Compatible taper-volume commercial system for Swietenia macrophylla King (mahogany) in Quintana Roo, Mexico}

\author{
Jonathan Hernández Ramos', Adrián Hernández Ramos²*, Xavier García Cuevas', Luis Martínez Ángel³, \\ Juan Carlos Tamarit Urías ${ }^{4}$ y Guadalupe Geraldine García Espinoza ${ }^{5}$
}

Instituto Nacional de Investigaciones Forestales, Agrícolas y Pecuarias. Campo Experimental Chetumal. Chetumal, Quintana Roo, México.

2 Instituto Nacional de Investigaciones Forestales, Agrícolas y Pecuarias. Campo Experimental Saltillo.

Saltillo, Coahuila, México.

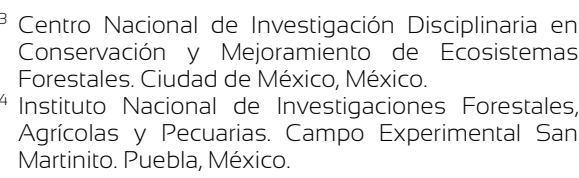

${ }^{3}$ Centro Nacional de Investigación Disciplinaria en Conservación y Mejoramiento de Ecosistemas Forestales. Ciudad de México, México.

${ }^{4}$ Instituto Nacional de Investigaciones Forestales, Agrícolas y Pecuarias. Campo Experimental San Martinito. Puebla, México.

\author{
5 Universidad Autónoma de Nuevo León. Linares, \\ Nuevo León, México. \\ * Autor de correspondencia. adrian.hernandezr90(a) \\ gmail.com
}

\section{RESUMEN}

La estimación del volumen comercial es importante para elaborar y ejecutar programas de manejo forestal. El conocer el perfil diamétrico del fuste y la distribución de productos maderables permite realizar evaluaciones financieras de la distribución de productos del arbolado comercial de manera objetiva. La información limitada sobre modelos y sistemas de cubicación para cuantificar volúmenes comerciales en masas arboladas tropicales conduce a plantear el objetivo de generar un sistema segmentado de ahusamiento (d) compatible con el volumen comercial $(V c)$ para Swietenia macrophylla King (caoba) en bosques tropicales de Quintana Roo. Se colectó una muestra de 116 árboles distribuida en la parte centro y sur del estado; con las variables de ahusamiento, volumen comercial y volumen total se ajustaron tres sistemas compatibles de $d-V c$ de tipo segmentado. El mejor ajuste estadístico lo presentó el sistema de Fang, al corregir problemas de heterocedasticidad y autocorrelación. Los parámetros $b_{0}, b_{1}$, y $b_{2}$ de este sistema corresponden al modelo de Schumacher-Hall para estimar el volumen total. Los puntos de cambio dendrométricos en el fuste se presentan en promedio a $5.6 \%$ y $83.1 \%$ de la altura total. El factor de forma determinado fue de 0.47 y el índice de esbeltez de 0.48 . El sistema de cubicación es una herramienta cuantitativa de apoyo en la elaboración de planes de manejo forestal para S. macrophylla.

PALABRAS CLAVE: bosque tropical, distribución de productos, perfil fustal, volumen maderable.

\section{ABSTRACT}

The volume estimation is important to develop and implement forest management plans. Therefore, knowing the profile of stem, and forest products distribution allows to obtain objective financial assessments of products distribution for commercial trees. The limited information available about models and volume systems to quantify the merchantable volumes in tropical forests, led the authors to consider the purpose of generating a compatible segmented system taper $(d)$ with merchantable volume $(V c)$ for Swietenia macrophylla King (Mahogany) in Quintana Roo tropical forests. Sample size was of 116 trees distributed in the central and southern parts of the state; with taper variables, commercial and total volume three compatible $d$ - $V c$ systems of the segmented type were adjusted. The best statistical fit was reached with the Fang system, which was readjusted correcting heterocedasticity and autocorrelation. The first three parameters of this system correspond to a Schumacher-Hall model employed to estimate total volume. Dendrometric shape change points in the trunk are located at 5.6\% and $83.1 \%$ in relation to total height. The form factor determined was 0.47 and slenderness ratio 0.48. Cubing system is a quantitative tool to support the development of forest management plans for S. macrophylla.

KEYWORDS: tropical forest, product distribution, stem profile, timber volume. 


\section{INTRODUCCIÓN}

El volumen es la variable más importante que se estima en los inventarios forestales. La predicción exacta del volumen fustal y comercial es fundamental para la planificación, evaluación y elaboración de programas de manejo con fines de aprovechamiento maderable (Torres y Magaña, 2001); sin embargo, en la mayoría de los casos existe cierto nivel de incertidumbre por parte de los dueños o poseedores de los bosques por conocer las existencias maderables en sus rodales (López, Cruz, Nájera y Hernández, 2015).

Los métodos utilizados para la cuantificación del volumen, son de tipo directo e indirecto; el primero requiere de un muestreo destructivo, cuyo costo y tiempo es elevado; el segundo se refiere a expresiones matemáticas que relacionan variables de diámetros y alturas comerciales para construir modelos tanto para describir el perfil fustal como para estimar el volumen total, el volumen comercial y obtener una distribución porcentual del volumen (Prodan, Peters, Cox, y Real, 1997). El conjunto de estos modelos por especie conforma lo que se denomina sistema de cubicación a nivel de árbol individual y constituye una herramienta poderosa de apoyo para el manejo forestal.

Las funciones de ahusamiento estiman el diámetro a cualquier altura comercial del árbol o viceversa, por integración matemática se genera una expresión que permite estimar el volumen comercial para determinados productos que se destinan a la industria forestal de acuerdo con su diámetro o altura comercial predefinidos (Kozak, 2004). Con la integración matemática se genera un sistema de ecuaciones compatibles $d-V_{c}$ que al compartir los mismos parámetros y una estructura geométrica similar puede ajustarse por regresión de manera simultánea, con esto, se logra reducir la varianza, por lo que se hace más consistente estadísticamente (Cruz-Cobos, De los SantosPosadas y Valdéz-Lazalde, 2008).

Las diferentes funciones de ahusamiento son definidas por sus expresiones matemáticas y pueden ser desde un polinomio simple, de exponente variable, trigonométrico, hasta uno de tipo segmentado; siendo mejores, en términos de precisión, estos últimos (Trincado y Burkhart, 2006). Por esta razón, para modelar el perfil diamétrico o fustal del arbolado en las masas forestales comerciales se han utilizado con mayor frecuencia las funciones de ahusamiento de tipo segmentadas compatibles con el volumen comercial; estas funciones además tienen flexibilidad para modelar tanto arboles de bosques naturales como de plantaciones forestales comerciales (Hernández, De los Santos, Ángeles, Valdez y Volke, 2013; Tamarit et al., 2014; Quiñonez-Barraza, De los Santos-Posadas, Álvarez-González y Velázquez-Martínez, 2014; UrangaValencia, De los Santos-Posadas, Valdez-Lazalde, LópezUpton y Navarro-Garza, 2015).

El volumen de un árbol, el factor de forma y la distribución de los productos están influenciados por las condiciones ambientales del rodal, por la densidad o por la silvicultura aplicada (Halord y Hocker, 1984; UrangaValencia et al., 2015). Por esto, la actualización de las herramientas silvícolas utilizadas en la elaboración y ejecución de programas de manejo forestal es fundamental para un manejo sostenible de los recursos. En Quintana Roo y la Península de Yucatán, las ecuaciones para estimar volumen requieren ser actualizadas, de esta forma, es posible obtener una valoración mejor de los productos forestales.

\section{OBJETIVOS}

El objetivo fue generar un sistema compatible de ahusamiento-volumen comercial, a partir del ajuste y evaluación de tres modelos de ahusamiento de tipo segmentados, ajustados en forma compatible con sus respectivas funciones de volumen comercial para Swietenia macrophylla King (caoba) en bosques tropicales de Quintana Roo. El sistema de cubicación será una herramienta de utilidad para construir tablas de volumen total y volumen comercial con distribución de productos, en la elaboración y ejecución de programas de manejo.

\section{MATERIALES Y MÉTODOS}

La muestra de árboles analizada se colectó en los ejidos de Felipe Carrillo Puerto, Chan Santa Cruz, Naranjal Poniente, X-Hazil, Bacalar y Caobas en el estado de Quintana Roo. El clima es cálido sub-húmedo (Am) y los suelos son de 
tipo Litosol y Rendzina con topoformas correspondientes a lomeríos bajos y llanuras rocosas (Comisión Nacional Forestal [Conafor], 2014).

El sistema de muestreo fue selectivo y se aplicó un método no destructivo mediante medición indirecta; en predios forestales bajo manejo se seleccionaron 116 árboles monopódicos, sin malformaciones, sin presencia de plagas o enfermedades y sin daños por incendios o rayos. La muestra abarcó la variabilidad de formas y tamaños de la especie en todas las categorías diamétricas. Se midieron de forma directa los diámetros del fuste a $0.3 \mathrm{~m}, 0.6 \mathrm{~m}, 0.9 \mathrm{~m}$ y $1.3 \mathrm{~m}$ de altura, este último corresponde al diámetro normal $(D)$, y luego a $2.5 \mathrm{~m}$ de altura, posteriormente, con un telerelascopio de Bitterlich Modelo CP: "Metrisch Correction Porcentaje ${ }^{\circledR}$ ", se midieron en forma indirecta los diámetros a la base del árbol $\left(d_{0}\right)$ y tanto los diámetros como las alturas a distintas secciones $\left(d_{i}\right.$ y $\left.H m_{i}\right)$ del fuste hasta llegar a la altura total $(H)$ y diámetro cero (punta del árbol). El empleo de este tipo de medición indirecta es una opción que permite hacer distribuciones y proyecciones de productos sin emplear un método destructivo (Kurinobu, Daryono, Mohanmad y Matsune, 2007)

El volumen de cada sección o troza se calculó con la fórmula de Smalian, el de la punta de cada árbol con la del cono y el volumen total $(V t)$ por árbol se obtuvo al utilizar el método de trozas traslapadas (Bailey, 1995). Con las variables dasométricas referidas, se conformó una base de datos de ahusamiento-volumen para su análisis y procesamiento estadístico.

En el análisis de regresión, se evaluaron tres sistemas compatibles de ahusamiento y volumen comercial $(d-V c)$ (Tabla 1); los dos primeros suponen la existencia de dos puntos de inflexión que indican el cambio de cuerpo dendrométrico en el fuste, en tanto que el último supone un solo punto de inflexión.

El ajuste simultáneo de cada sistema compatible se realizó con el programa estadístico SAS $9.2 \AA$, mediante el procedimiento model y la técnica de máxima verosimilitud con información completa (fiml) de SAS (SAS Institute Inc., 2009). El ajuste con fiml produce estimaciones consistentes en los parámetros cuando el ajuste se realiza de forma simultánea, lo cual minimiza los errores de ambas ecuaciones $d$ - Vc (Fang et al., 2000; Cruz-Cobos et al., 2008). Para evitar problemas de convergencia al estimar los parámetros cuando $H c_{i}=H$, es decir $d_{i}=0$, se agregó un valor de delta igual a 0.01 en este punto y una variable indicadora en la punta del árbol (Fang et al., 2000).

En estudios de ahusamiento-volumen las variables analizadas tienen múltiples observaciones en una misma unidad de muestreo (fuste del árbol), lo cual viola algunos supuestos de la teoría de regresión, básicamente se presentan problemas de heterocedasticidad y autocorrelación de los errores, situación que ocasiona parámetros ineficientes y errores estándar mayores al mínimo buscado (Hernández et al., 2013). La heterocedasticidad se corrigió con regresión ponderada, la cual proporcionó varianza menor $\left(1 /\left(D^{2} A\right)\right.$ ) (Fernández, Fassola y García, 2011); la autocorrelación se corrigió mediante un modelo autorregresivo de tiempo continuo (CAR(X)) (Zimmerman y Nunez-Anton, 2001; Rojo, Perales, Sánchez-Rodríguez, Álvarez-González y Gadow, 2005).

La evaluación de la calidad de ajuste y selección del mejor sistema se realizó con base en los valores menores de la suma de cuadrados del error (SCE) y de la raíz del cuadrado medio del error (RCME); el valor mayor en el coeficiente de determinación ajustado $\left(R_{a j}^{2}\right)$, además de la significancia de los parámetros a un nivel de confiabilidad de 95\% (Corral, Bario, Aguirre y Diéguez, 2007). La homocedasticidad se verificó de forma gráfica y la autocorrelación se evaluó con la prueba de Durbin-Watson (DW) (Da Cunha, Vargas y Escalier, 2009).

Con el mejor sistema seleccionado, se determinó el factor de forma promedio del fuste, al dividir el volumen que estima el sistema y el volumen de un cilindro teórico, de acuerdo con las variables diámetro normal y altura total; esto se realizó por árbol y se obtuvo un promedio. También se calculó el índice de esbeltez para cada categoría diamétrica, tal como lo sugieren Nájera-Luna y HernándezHernández (2008). Finalmente, con las estimaciones realizadas con el sistema compatible seleccionado, se construyó una tabla de distribución de productos. 
Tabla 1. Sistemas compatibles de ahusamiento-volumen comercial $(d-V c)$ de tipo segmentado analizados para caoba en bosques tropicales de Quintana Roo.

No.

Sistema compatible

\section{Fang, Borders y Bailey (2000)}

$d=c_{1}\left[H^{\frac{K-\beta_{1}}{\beta_{1}}}(1-q)^{\frac{K-\beta_{1}}{\beta_{1}}} \alpha_{1}^{l_{1}+I_{2}} \alpha_{2}^{l_{2}}\right]^{1 / 2}\left\{\begin{array}{l}l_{1}=1 \text { si } p_{1} \leq q \leq p_{2} ; \text { de lo contrario } O \\ l_{2}=1 \text { si } p_{2} \leq q \leq 1 ; \text { de lo contrario } O\end{array}\right.$

$\left.R_{0}=\left(1-H_{b} / H\right)^{K / \beta_{1}} ; R_{1}=\left(1-P_{1}\right)^{K / \beta_{1}} ; R_{2}=\left(1-P_{2}\right)^{K / \beta_{2}} ; \beta=\beta_{1}^{1-\left(l_{1}+I_{2}\right)} \beta_{2}^{I_{1}} \beta_{3}^{I_{2}} ;\right\}$

(1)

$$
\begin{aligned}
C_{1} & =\sqrt{\frac{b_{0} D^{b_{1}} H^{b_{2}-\frac{k}{\beta_{1}}}}{\beta_{1}\left(R_{0}-R_{1}\right)+\beta_{2}\left(R_{1}-\alpha_{1} R_{2}\right)+\beta_{3} \alpha_{1} R_{2}}} \\
\alpha_{1} & =\left(1-p_{1}\right)^{\frac{K\left(\beta_{2}-\beta_{1}\right)}{\beta_{1} \beta_{2}}} ; \alpha_{2}=\left(1-p_{2}\right)^{\frac{K\left(\beta_{3}-\beta_{2}\right)}{\beta_{2} \beta_{3}}} \\
V_{c} & =C_{1}^{2} H^{\frac{k}{\beta_{1}}}\left[\beta_{1} R_{0}+\left(I_{1}+I_{2}\right)+\left(\beta_{2}-\beta_{1}\right) R_{1}+I_{2}\left(\beta_{3}-\beta_{2}\right) \alpha_{1} R_{2}-\beta(1-q)^{\frac{k}{\beta}} \alpha_{1}^{I_{1}+I_{2}} \alpha_{2}^{I_{2}}\right]
\end{aligned}
$$

\section{Max y Burkhart (1976)}

$$
d=D\left[\beta_{1}(q-1)+\beta_{2}\left(q^{2}-1\right)+\beta_{3}\left(p_{1}-q\right)^{2} I_{1}+\beta_{4}\left(P_{2}-q\right)^{2} I_{2}\right]^{1 / 2} \quad\left\{\begin{array}{l}
l_{1}=1 \text { si } q \leq p_{1} ; \text { de lo contrario } 0 \\
l_{2}=1 \text { si } q \leq P_{2} ; \text { de lo contrario } 0
\end{array}\right.
$$

(2)

$$
V_{c}=k D^{2} H\left\{\frac{\beta_{2}}{3}\left(q^{3}-z_{1}^{3}\right)+\frac{\beta_{1}}{2}\left(q^{2}-z_{1}^{2}\right)-\left(\beta_{1}+\beta_{2}\right)\left(q-z_{1}\right)-\frac{\beta_{3}}{3}\left[\left(p_{1}-q\right)^{3} J_{1}-\left(p_{1}-z_{1}\right)^{3} K_{1}\right]-\frac{\beta_{4}}{3}\left[\left(p_{2}-q\right)^{3} J_{2}-\left(p_{2}-\right.\right.\right.
$$

$\left.\left.\left.z_{1}\right)^{3} K_{2}\right]\right\}$ donde $\left\{\begin{array}{c}J_{1}=1 \text { si } q \leq P_{1} ; J_{2}=O \text { si } q>P_{2} \\ K_{1}=1 \text { si } z_{1} \leq P_{1} ; K_{2}=O \text { si } z_{1}>P_{1}\end{array}\right.$

\section{Parresol, Hotvedt y Cao (1987)}

(3)

$$
\begin{aligned}
& d=D\left[Z^{2}\left(\beta_{1}+\beta_{2} Z\right)+\left(Z-p_{1}\right)^{2}\left(\beta_{3}+\beta_{4}\left(Z+2 p_{1}\right) I_{1}\right)\right]^{1 / 2} V_{c}=k D^{2} H\left\{\left[\left(\frac{\beta_{2}+\beta_{4} l_{1}}{4\left(Z^{4}-Z_{b}^{4}\right)}\right)+\left(\frac{\beta_{1}+\beta_{3} l_{1}}{3\left(Z^{3}-z_{b}^{3}\right)}\right)+(\right.\right. \\
& \left.\left.\left.\frac{2 \beta_{1}\left(p_{1} l_{1}\right)+\left(2 p_{1} \beta_{4}\right)}{2\left(Z^{2}-Z_{b}^{2}\right)}\right)+\left(\left(p_{1}^{2} l_{1} \beta_{3}\right)+\left(2 p_{1}-\beta_{4}\right)\left(Z-Z_{b}\right)\right)\right]\right\} \quad \text { donde } I_{1}=1 \text { si } Z \geq p_{1} ; \text { de lo contrario } 0
\end{aligned}
$$

D: diámetro normal (cm), d: diámetro (cm) a la altura Hm (m) desde el nivel del tocón, H: altura total (m), Hb: altura del tocón (m), $V_{c}$ : volumen comercial $\left(\mathrm{m}^{3}\right)$, R: constante $=\pi / 40$ 000, li: vaiables indicadoras de cambios dendrométricos en el fuste del árbol. $\alpha_{i}$, bi, $\beta_{i}$ y pi son parámetros a ser estimados.

\section{ReSUltadOS}

El ajuste de los tres sistemas se realizó con las correcciones de heterocedasticidad en ambas funciones (ahusamientovolumen) y de la autocorrelación al usar un modelo autorregresivo de orden CAR (3) (Tabla 2). Los sistemas ajustados con la estructura CAR (3) presentaron parámetros significativos a $95 \%(\mathrm{p}=0.05)$ y los valores menores en los estadísticos $S C E$ y $R C M E$, y $\mathrm{R}_{a j}^{2}$ superior a 0.89 .

Los ajustes de los tres sistemas compatibles fueron adecuados para modelar el perfil fustal y para estimar el volumen comercial y total, sin embargo, el mejor sistema $d$ $V c$ fue el de Fang et al. (2000), porque comparativamente 
presentó los valores más bajos en la SCE y RCME, y los valores mayores en $\mathrm{R}_{a j}^{2}$, al explicar $97 \%$ y $99 \%$ de la variabilidad del perfil fustal y del volumen comercial, respectivamente. Las estimaciones realizadas con el sistema de Fang et al. (2000) no evidenciaron heterocedasticidad en ambos componentes $d-V_{c}$ (Fig. 1) y presentaron los mejores valores en el estadístico de $D W$ (Tabla 2).
Los valores de los parámetros $b_{i}$ obtenidos en el sistema de Fang et al. (2000) corresponden al modelo de volumen total de Schumacher-Hall: $V t=0.000071 D^{1.940562}$ $H^{0.831416}$. El factor de forma (ff) promedio obtenido para los arboles de caoba en Quintana Roo fue de 0.47, cercano a un paraboloide (Prodan et al., 1997). El índice de esbeltez promedio fue de 0.48 y disminuyó a medida que el árbol incrementaba sus dimensiones (Tabla 3).

Tabla 2. Ajuste estadístico y valores de los parámetros para los tres sistemas compatibles ajustados a árboles de caoba en Quintana Roo.

\begin{tabular}{|c|c|c|c|c|c|c|c|c|c|c|}
\hline Sistema & Variable & SCE & RCME & $R^{2} a j$ & $D W$ & Parámetro & Estimación & Eea & $\begin{array}{c}\text { Valor } \\
\mathrm{t}\end{array}$ & $\operatorname{Pr}>|t|$ \\
\hline \multirow[t]{11}{*}{ (1) } & $V c$ & 1.5844 & 0.0397 & 0.995 & 1.7 & $b_{0}$ & 0.000071 & 6.13E-06 & 11.56 & $<0.0001$ \\
\hline & $d$ & 8154.1 & 2.8477 & 0.974 & 1.5 & $b_{1}$ & 1.940562 & 0.021 & 92.25 & $<0.0001$ \\
\hline & & & & & & $b_{2}$ & 0.831416 & 0.0363 & 22.92 & $<0.0001$ \\
\hline & & & & & & $\beta_{1}$ & 1.00E-05 & 8.03E-07 & 12.71 & $<0.0001$ \\
\hline & & & & & & $\beta_{2}$ & 0.000032 & 3.32E-07 & 96.53 & $<0.0001$ \\
\hline & & & & & & $\beta_{3}$ & 0.000053 & 5.93E-06 & 8.99 & $<0.0001$ \\
\hline & & & & & & $p_{1}$ & 0.055977 & 0.00365 & 15.34 & $<0.0001$ \\
\hline & & & & & & $p_{2}$ & 0.831412 & 0.019 & 43.85 & $<0.0001$ \\
\hline & & & & & & pld & 1.087964 & 0.0134 & 81.04 & $<0.0001$ \\
\hline & & & & & & $p 2 d$ & 1.043224 & 0.0123 & 84.88 & $<0.0001$ \\
\hline & & & & & & $p 3 d$ & 1.014956 & 0.0147 & 69.27 & $<0.0001$ \\
\hline \multirow[t]{9}{*}{ (2) } & $V c$ & 4.2163 & 0.0646 & 0.986 & 0.7 & $\beta_{1}$ & -2.82087 & 0.5242 & -5.38 & $<0.0001$ \\
\hline & $d$ & 9657.4 & 3.093 & 0.970 & 2.5 & $\beta_{2}$ & 1.273498 & 0.2938 & 4.33 & $<0.0001$ \\
\hline & & & & & & $\beta_{3}$ & 130.2544 & 23.8329 & 5.47 & $<0.0001$ \\
\hline & & & & & & $\beta_{4}$ & -0.8953 & 0.2723 & -3.29 & 0.001 \\
\hline & & & & & & $p_{1}$ & 0.059294 & 0.0043 & 13.8 & $<0.0001$ \\
\hline & & & & & & $p_{2}$ & 0.725046 & 0.0834 & 8.7 & $<0.0001$ \\
\hline & & & & & & pld & 0.829826 & 0.0146 & 56.88 & $<0.0001$ \\
\hline & & & & & & $p 2 d$ & 0.894429 & 0.0107 & 83.89 & $<0.0001$ \\
\hline & & & & & & $p 3 d$ & 0.6054 & 0.0326 & 18.55 & $<0.0001$ \\
\hline \multirow[t]{8}{*}{ (3) } & $V c$ & 30.131 & 0.1726 & 0.899 & 1.4 & $\beta_{1}$ & 2.09367 & 0.1037 & 20.18 & $<0.0001$ \\
\hline & $d$ & 10266 & 3.1866 & 0.968 & 2.2 & $\beta_{2}$ & -1.03686 & 0.1766 & -5.87 & $<0.0001$ \\
\hline & & & & & & $\beta_{3}$ & -0.56032 & 0.149 & -3.76 & $<0.0001$ \\
\hline & & & & & & $\beta_{4}$ & 1.409235 & 0.1822 & 7.74 & $<0.0001$ \\
\hline & & & & & & $p_{1}$ & 0.694987 & 0.0911 & 7.63 & $<0.0001$ \\
\hline & & & & & & pld & 0.722322 & 0.0243 & 29.67 & $<0.0001$ \\
\hline & & & & & & $p 2 d$ & 0.581341 & 0.0493 & 11.8 & $<0.0001$ \\
\hline & & & & & & p3d & 0.419604 & 0.0513 & 8.18 & $<0.0001$ \\
\hline
\end{tabular}

Eea: error estándar aproximado. $b_{i} ; \beta_{i^{\prime}} p_{1}$ y $p_{2}$ : parámetros estimados. pld, p2d y p3d: parámetros del modelo autoregresivo CAR (3). 

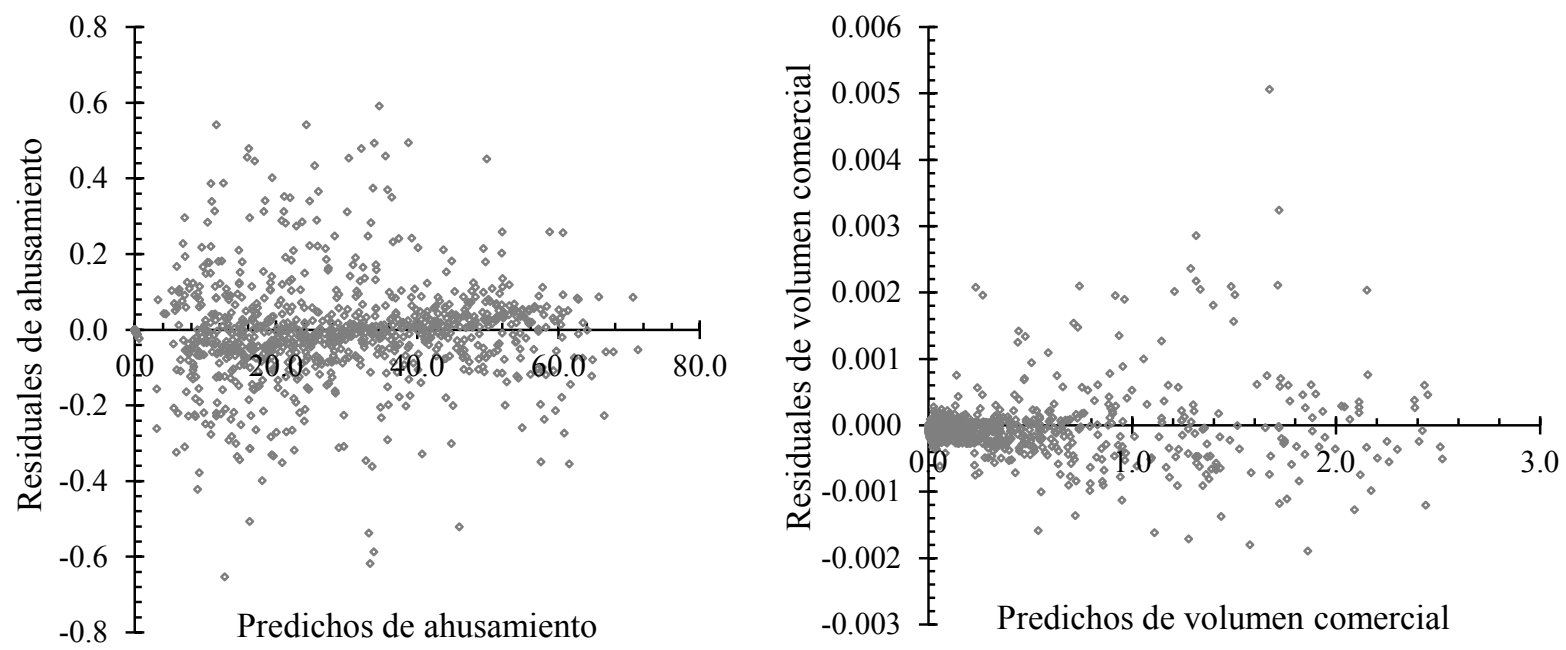

Figura 1. Comportamiento gráfico de los residuales del ahusamiento (izquierda) y del volumen comercial (derecha) del sistema compatible de Fang et al. (2000) con tendencia homocedástica.

Tabla 3. Índice de Esbeltez (IE) por categoría diamétrica $(C D)$ determinado para árboles de caoba en Quintana Roo.

\begin{tabular}{|c|c|c|c|c|c|c|c|c|c|c|c|c|}
\hline$C D$ & 10 & 15 & 20 & 25 & 30 & 35 & 40 & 45 & 50 & 55 & 60 & 65 \\
\hline IE & 0.95 & 0.77 & 0.63 & 0.60 & 0.57 & 0.51 & 0.45 & 0.42 & 0.36 & 0.34 & 0.35 & 0.30 \\
\hline
\end{tabular}

El sistema compatible seleccionado estima, en promedio, el primer punto de inflexión en los árboles de caoba a 5.6\% $\left(p_{1}\right)$ de la altura total y es donde sucede el cambio de cuerpo dendrométrico de neiloide a paraboloide, mientras que el cambio de paraboloide a cono sucede a $83.1 \%\left(p_{2}\right)$ de la altura del árbol (Tabla 2).

$\mathrm{Al}$ verificar las estimaciones obtenidas con el sistema de Fang et al. (2000), se observó un comportamiento lógico porque el diámetro disminuye y el volumen comercial se acumula progresivamente a medida que la altura en el fuste aumenta (Fig. 2).

La tabla de distribución de productos para arboles individuales de caoba que se construyó con el sistema compatible seleccionado se muestra en la tabla 4. En su elaboración se establecieron los diámetros comerciales siguientes: para productos primarios (chapa y aserrío), diámetros de $32.5 \mathrm{~cm}$ o superiores; para productos secundarios (cortas dimensiones) de $17.5 \mathrm{~cm}$ a $32.4 \mathrm{~cm}$; y de $17.5 \mathrm{~cm}$ o menores para productos como celulósicos y leña (Tabla 4).

El sistema compatible $d$ - $V c$ de Fang et al. (2000) estima con precisión el volumen total y comercial en árboles de caoba; por ejemplo, para un árbol promedio con $55 \mathrm{~cm}$ de diámetro normal y $20 \mathrm{~m}$ de altura total, se estima un volumen total de $2.0429 \mathrm{~m}^{3}$ (al aplicar el modelo de $V t=0.000071 D^{1.940562} H^{0.831416}$ ). En su respectiva distribución de productos que se destinaría a la industria forestal, se tendría que el mayor volumen se concentra para productos primarios (trocería comercial) con $1.707 \mathrm{~m}^{3}$; para productos secundarios con $0.2806 \mathrm{~m}^{3}$; y para celulosa y leña se estima un volumen mínimo con valor de $0.0555 \mathrm{~m}^{3}$ (Tabla 4).

Lo anterior permite obtener una valoración económica por tipo de producto, de acuerdo con los precios de los productos maderables para caoba señalados por la Secretaría de Medio Ambiente y Recursos Naturales y la 

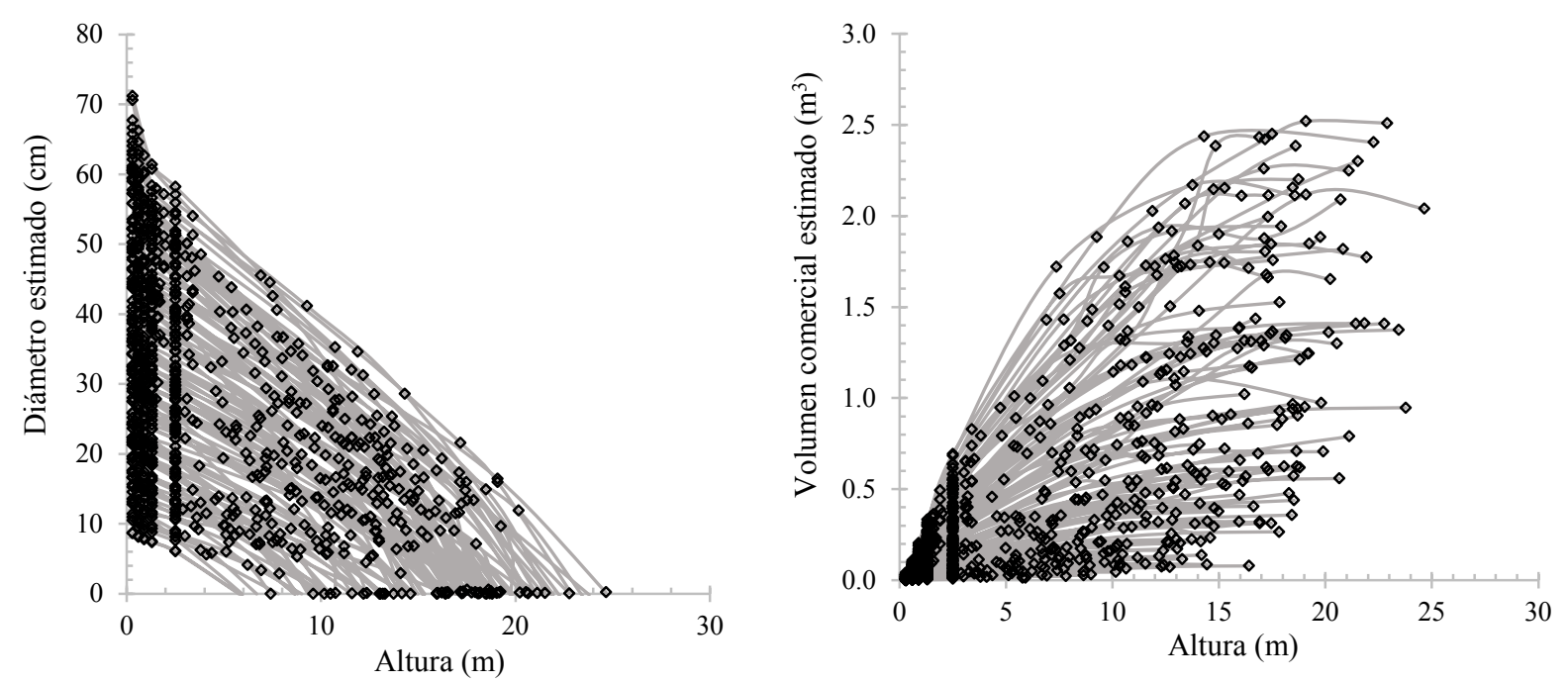

Figura 2. Distribución del diámetro (izquierda) y del volumen comercial (derecha) estimados con el sistema $d$ - $V c$ de Fang et al. (2000) respecto a la altura $(\mathrm{Hm})$ sobre el fuste para árboles de caoba.

Tabla 4. Tabla de volumen con distribución de productos para arboles individuales de caoba en Quintana Roo.

\begin{tabular}{|c|c|c|c|c|c|c|c|c|c|c|c|}
\hline \multirow{4}{*}{$\begin{array}{c}C D \\
(\mathrm{~cm})\end{array}$} & \multicolumn{11}{|c|}{ Altura total } \\
\hline & \multicolumn{2}{|c|}{$10 \mathrm{~m}$} & \multicolumn{3}{|c|}{$15 \mathrm{~m}$} & \multicolumn{3}{|c|}{$20 \mathrm{~m}$} & \multicolumn{3}{|c|}{$25 \mathrm{~m}$} \\
\hline & $\begin{array}{l}\text { Cel. y } \\
\text { leña }\end{array}$ & Sec. & $\begin{array}{l}\text { Cel. y } \\
\text { leña }\end{array}$ & Sec. & Com. & $\begin{array}{l}\text { Cel. y } \\
\text { leña }\end{array}$ & Sec. & Com. & $\begin{array}{l}\text { Cel. y } \\
\text { leña }\end{array}$ & Sec. & Com. \\
\hline & \multicolumn{11}{|c|}{ Metros cúbicos $\left(\mathrm{m}^{3}\right)$} \\
\hline 10 & 0.0420 & & & & & & & & & & \\
\hline 15 & 0.0922 & & 0.1292 & & & & & & & & \\
\hline 20 & 0.0696 & 0.0916 & 0.1099 & 0.1159 & & & & & & & \\
\hline 25 & & & 0.0831 & 0.2651 & & 0.1146 & 0.3277 & & & & \\
\hline 30 & & & 0.0670 & 0.4291 & & 0.0922 & 0.5379 & & & & \\
\hline 35 & & & 0.0567 & 0.3706 & 0.2417 & 0.0778 & 0.5128 & 0.2592 & 0.0994 & 0.6592 & 0.2644 \\
\hline 40 & & & 0.0500 & 0.3101 & 0.5068 & 0.0683 & 0.4291 & 0.6038 & 0.0870 & 0.5516 & 0.6870 \\
\hline 45 & & & 0.0456 & 0.2650 & 0.7789 & 0.0620 & 0.3667 & 0.9553 & 0.0787 & 0.4714 & 1.1159 \\
\hline 50 & & & 0.0428 & 0.2303 & 1.0636 & 0.0579 & 0.3186 & 1.3214 & 0.0733 & 0.4096 & 1.5612 \\
\hline 55 & & & 0.0412 & 0.2028 & 1.3643 & 0.0555 & 0.2806 & 1.7068 & 0.0699 & 0.3607 & 2.0287 \\
\hline 60 & & & 0.0406 & 0.1805 & 1.6830 & 0.0543 & 0.2498 & 2.1145 & 0.0681 & 0.3211 & 2.5224 \\
\hline 65 & & & 0.0407 & 0.1623 & 2.0211 & 0.0507 & 0.2279 & 2.5464 & 0.0676 & 0.2886 & 3.0447 \\
\hline
\end{tabular}

CD: Categoría diametrica, Cel. y leña: Celulósicos y leña, Sec: Cortas dimensiones, Com: Primarios.

Conafor [Semarnat-Conafor] (2016) para el segundo trimestre del año 2016. El valor de producto primario para el árbol del ejemplo es de MXN 4077.85 y el de secundarios de MXN 1472.22, en tanto que la leña en la zona de estudio se ocupa para consumo local y no tiene valor económico para la industria, por tanto, no se consideró para la 
proyección económica. De esta manera, para un árbol de 55 $\mathrm{cm}$ de diámetro normal y $20 \mathrm{~m}$ de altura total se estiman un valor total de MXN 7373.18, donde $94.3 \%$ se obtiene con trocería comercial y $5.6 \%$ con trocería secundaria.

\section{DISCUSIÓN}

Los resultados obtenidos al utilizar un modelo de orden CAR(3) en los sistemas compatibles de ahusamientovolumen comercial evaluados, son similares a los publicados por Milena, Barrios y Trincado (2015), quienes utilizan en los modelos de ahusamiento una estructura autorregresiva de orden 3 en Eucalyptus tereticornis y los resultados estadísticos mejores se obtuvieron con los modelos mencionados, debido a que los modelos autorregresivos de orden 1 y 2 no reducen adecuadamente la autocorrelación de los errores en el sistema.

El sistema $d-V c$ segmentado compatible de Fang et al. (2000) presentó los estadísticos de ajuste mejores, este sistema ha mostrado resultados similares en otros estudios, por ejemplo en bosques manejados de Pinus patula en Hidalgo, México (Hernández et al., 2013); en seis especies de bosques naturales del género Pinus en Durango, México (Quiñonez-Barraza et al., 2014); al comparar la forma y el rendimiento maderable de $P$. patula en bosques naturales en los estados de Hidalgo y Oaxaca (Uranga-Valencia et al., 2015), México; y al emplear estos mismos modelos para construir sistemas compatibles en bosques de Pinus ayacabuite en Oaxaca (Ramírez-Martínez, Santiago-García, Quiñonez-Barraza, Ruiz-Aquino y Rodríguez-Ortiz, 2016).

La regresión ponderada utilizada redujo la varianza en los modelos que conforman el sistema compatible y presentó una distribución homogénea de los residuales, lo cual de acuerdo con Fernández et al. (2011) no evidenció problemas de heterocedasticidad. Con base en Rojo et al. (2005) y Da Cunha et al. (2009), el estadístico de $D W$ con valor $\geq 1.5$ indica que no existe correlación espacial entre los datos y que los errores son independientes.

El sistema compatible propuesto tiene una capacidad alta para describir el perfil fustal y estimar el volumen comercial; además del volumen total. Con este sistema de cubicación se actualizan los modelos que hasta ahora siguen siendo utilizados para elaborar y ejecutar programas de manejo forestal y que fueron generados para diferentes especies tropicales maderables por De los Santos (1976) para la Península de Yucatán y por Patiño, López y Gómez (1994) bajo condiciones ambientales y físicas diferentes a las actuales.

El factor de forma promedio determinado para la muestra de árboles de esta especie fue de 0.47 , lo cual pone de manifiesto que han sobreestimado los volúmenes fustales aprovechados en los programas de manejo debido a que, para las dos ecuaciones propuestas por De los Santos (1976), se estima un ff de 0.80 y 0.72 , y de 0.57 para los modelos señalados por Patiño et al. (1994). El valor menor del ff calculado en este estudio puede probablemente ser atribuido al cambio de las condiciones de la composición, estructura y manejo silvícola de los bosques tropicales de Quintana Roo, en donde las dimensiones del arbolado de esta especie se han reducido, debido a un sobre aprovechamiento o a un manejo inadecuado de estos bosques.

El índice de esbeltez (IE) promedio obtenido, de 0.48, indica mayor estabilidad en los árboles ante los efectos de vientos fuertes. Esto coincide con Arias (2004), quien refiere que $I E$ bajos son más estables ante daños mecánicos por la asociación de la forma geométrica que los individuos tienen, mientras que individuos con IE altos (1:1 o superiores) son menos estables (Durlo, Jaques y Denardi, 1998). El valor de $I E$ muestra una relación proporcional de crecimiento promedio en los árboles de la especie donde, por cada centímetro que aumentan en diámetro normal, aumenta 0.48 metros de altura total.

Los puntos de inflexión encontrados en los árboles de caoba $(5.6 \%$ y $83.1 \%$ de la altura total) son similares a los hallados por López et al. (2015) con el sistema de Fang et al. (2000) en Pinus douglasiana, donde el primer y segundo punto de inflexión ocurrieron a $5.4 \%$ y $66.8 \%$ de la altura total, respectivamente; sin embargo, difiere con los resultados de Milena et al. (2015) que van desde 3.9\% hasta $48.0 \%$ para el primer punto de inflexión y de $80.0 \%$ a $96.2 \%$ para el segundo punto, al utilizar los modelos segmentados de Max y Burkhart (1976); Cao, Burkhart y Max (1980) y 
Parresol et al. (1987) en E. tereticornis. Esta diferencia puede ser atribuible a las especies, tasas de crecimiento, calidades de estación, densidad o los tratamientos silvícolas utilizados.

Las modelaciones del perfil fustal y las estimaciones del volumen comercial y total obtenidas con el sistema de Fang et al. (2000) presentan un comportamiento lógico y el patrón es similar al que encontraron Li y Weiskittel (2010) en Canadá para las principales especies de coníferas, y también a los hallados por Tamarit et al. (2014) en México para plantaciones de Tectona grandis (teca). El sistema de cubicación generado a partir de un modelo de ahusamiento que es compatible con su respectiva función de volumen comercial es una herramienta cuantitativa importante para la planeación del manejo técnico de los bosques donde esta especie se desarrolla, punto de vista que coincide con lo referido por Tapia y Návar (2011), por Santiago-García, De los Santos-Posadas, Ángeles-Pérez, Valdez-Lazalde y Ramírez-Valverde (2013) y por López et al. (2015), en el sentido de que los sistemas compatibles de ahusamiento y volumen comercial, juegan un papel importante en la planeación, evaluación y aprovechamiento forestal.

\section{CONCLUSIONES}

El sistema compatible de ahusamiento y volumen comercial de Fang et al. (2000) presentó la mejor calidad de ajuste de entre los sistemas evaluados y fue seleccionado como el mejor para describir de forma confiable y precisa el perfil fustal y para estimar el volumen comercial de los árboles de S. macrophylla. Este sistema contiene en forma explícita al modelo de volumen total de Schumacher-Hall.

El sistema de cubicación generado es una herramienta cuantitativa de alta utilidad por los prestadores de servicios técnicos forestales en la planeación y ejecución de los programas de manejo forestal en los bosques tropicales, porque S. macrophylla es una de las especies guía para la aplicación de sistemas silvícolas en la región, además permite realizar una valoración financiera de los productos a obtener de un aprovechamiento forestal maderable con esta especie. Esta herramienta puede integrarse como un paquete tecnológico y complementarse con información de inventarios forestales operativos para obtener las existencias maderables por tipos de productos para cada unidad de manejo.

\section{RECONOCIMIENTOS}

Esta investigación fue financiada por el gobierno del estado de Quintana Roo a través del Instituto Forestal (Infoqroo), por medio del proyecto "Estudio para realizar acciones de tablas de volúmenes de 8 especies forestales maderables". SINCOP-INIFAP 232179Y.

\section{REFERENCIAS}

Arias, D. (2004). Estudio de las relaciones altura-diámetro para seis especies maderables utilizadas en programas de reforestación en la Zona Sur de Costa Rica. Revista Forestal Kurú, 1(2), 1-11.

Bailey, L. R. (1995). Upper stem volumes from stem analysis data: an overlapping bolts method. Canadian Journal of Forest Research, 25(1), 170-173. doi: 10.1139/x95-020

Cao, Q. V., Burkhart, H. E., \& Max, T. A, (1980). Evaluating of two methods for cubic-volume prediction of loblolly pine to any merchantable limit. Forest Science, 26, 71-80.

Comisión Nacional Forestal [Conafor] (2014). Inventario nacional forestal y de suelos-Quintana Roo 2013. Guadalajara, Jalisco, México: Conafor.

Corral-Rivas, J. J., Bario, A. M., Aguirre C., A. O., \& Diéguez A., U. (2007). Use of stump diameter to estimate diameter at breast height and tree volume for major pine species in El Salto, Durango (México). Forestry, 80, 29-40. doi: 10.1093/forestry/cpl048

Cruz-Cobos, F., De los Santos-Posadas, H. M., \& Valdéz-Lazalde, J. R. (2008). Sistema compatible de ahusamiento-volumen para Pinus cooperi Blanco en Durango México. Agrociencia, 42(4), 473-485.

Da Cunha, T. A., Vargas M., J. O., \& Escalier, H. M. (2009). Ajuste y selección de modelos de regresión para estimar volumen total de árboles. Doc. Técnico 5-2009/FOMABO. Cochabamba, Bolivia: Escuela de Ciencias Forestales de la Universidad Mayor de San Simón.

De los Santos, V. M. (1976). Tablas de volumen para montes de la Peninsula de Yucatán. Tesis de Licenciatura. Universidad Autónoma Chapingo. Chapingo, Méx.

Durlo, A. M., Jaques, S. F, \& Denardi, L. (1998). Modelagem da copa de Cebrela fissilis Vellozo. Ciencia Florestal, 2(14), 79-89.

Fang, Z., Borders, B. E., \& Bailey, R. L. (2000). Compatible volumetaper models for loblolly and slash pine based on a system with 
segmented-stem form factors. Forest Science, 46, 1-12. doi: $10.1093 /$ forestscience/46.1.1

Harold, D., \& Hocker, J. (1984). Introducción a la biología forestal. F. A. Bellomo L. (Trad). México, D. F.: AGT Editor.

Hernández, P., D., De los Santos P., H. M., Ángeles P., G., Valdez L., J. R., \& Volke, H. V. H. (2013). Funciones de ahusamiento y volumen comercial para Pinus patula Schltdl. et Cham. en Zacualtipán, Hidalgo. Revista Mexicana de Ciencias Forestales, 4(16), $34-45$.

Kozak, A. (2004). My last words on taper equations. The Forestry Chronicle, 80(4), 507-515. doi: 10.5558/tfc80507-4

Kurinobu, S., Daryono, P., Mohanmad, N., \& Matsune, K. (2007). A stem taper equation compatible to volume equation for Paraserianthes falcataria in Pare, East Java, Indonesia: its implications for the plantation management. Journal of Forest Research, 12, 473-478. doi: 10.1007/s10310-007-0037-5

Li, R., \& Weiskittel, A. R. (2010). Comparison of model forms for estimating for stem taper and volume in the primary conifer species of the North American Acadian Region. Annals of Forest Science, 67(3), 302. doi: 10.1051/forest/2009109

López M., J. C., Cruz C., F., Nájera L., J. A., \& Hernández, F. J. (2015). Modelo de ahusamiento y volumen comercial para Pinus oocarpa y Pinus douglasiana en la región de Pueblo Nuevo, Durango. Investigación y Ciencia, 23(64), 47-53.

Max, T. A., \& Burkhart, H. E. (1976). Segmented polynomial regression applied to taper equations. Forest Science, 22, 283-289. doi: 10.1093/forestscience/22.3.283

Milena L., A., Barrios, A., \& Trincado, G. (2015). Modelos de perfiles fustales con una estructura de error autorregresivo para plantaciones de Eucalyptus tereticornis en Colombia. Madera $y$ Bosques, 21(2), 73-88. doi: 10.21829/myb.2015.212446

Nájera-Luna., J. A., \& Hernández-Hernández, E. (2008). Relaciones morfométricas de un bosque coetáneo de la Región de El Santo, Durango. Ra-Xambai, 4(1), 69-81.

Parresol, B. R., Hotvedt, J. E., \& Cao, Q. V. (1987). A volume and taper prediction system for bald cypress. Canadian Journal of Forest Research, 17, 250-259. doi: 10.1139/x87-042

Patiño V., F., López T., J. L., \& Gómez D. A. (1994). Selva (Versión 4) Paquete de cómputo para procesar datos de inventarios forestales para especies de la Península de Yucatán. Mérida, Yucatán: Instituto Nacional de Investigaciones Forestales y Agropecuarias.

Prodan, M., Peters, R., Cox, F., \& Real, P. (1997). Mensura forestal. Serie investigación y educación en desarrollo sostenible. San José, Costa Rica: Instituto Internacional de Cooperación para la
Agricultura (IICA)- Deutsche Gesellschaft füt Technische Zusammenarbeit (GTZ).

Quiñonez-Barraza, G., De los Santos-Posadas, H. M., ÁlvarezGonzález, J. G., \& Velázquez-Martínez, A. (2014). Sistema compatible de ahusamiento y volumen comercial para las principales especies de Pinus en Durango, México. Agrociencia, 48, 553-567.

Ramírez-Martínez, A., Santiago-García, W., Quiñonez-Barraza, G., RuizAquino, F., \& Rodríguez-Ortiz, G. (2016). Sistema compatible segmentado de ahusamiento-volumen comercial para Pinus ayacabuite Ehren. En F. Gallardo-López (Ed.). (2016). Innovando el agro veracruzano 2016. Frente a los retos de la relación Sociedad-Naturaleza (pp. 649-670). Veracruz, México: Colegio de Postgraduados.

Rojo, A., Perales, X., Sánchez-Rodríguez, F., Álvarez-González, J. G., \& Gadow, K. V. (2005). Stem taper functions for maritime pine (Pinus pinaster Ait.) in Galicia (Northwestern Spain). European Journal of Forest Research, 124, 177-186. doi: 10.1007/s10342-0050066-6

Santiago-García, W., De los Santos-Posadas, H. M., Ángeles-Pérez, G., Valdez-Lazalde, J. R., \& Ramírez-Valverde, G. (2013). Sistema compatible de crecimiento y rendimiento para rodales coetáneos de Pinus patula. Revista Fitotecnia Mexicana, 36(2), 163-172.

Secretaría de Medio Ambiente y Recursos Naturales [Semarnat]Conafor, (2016). Reporte de productos forestales maderables. Reporte trimestral abril/junio de 2016. Gerencia de Fomento a la Productividad Forestal Sustentable. Zapopan, Jalisco, México. Recuperado de http://www.cnf.gob.mx:8090/snif/portal/economica/sipre

SAS Institute Inc., (2009). SAS/STAT® 9.2 User's Guide. SAS Institute Inc. Raleigh, NC, EUA, autor.

Tamarit U., J. C., De los Santos P., H. M., Aldrete, A., Valdez L., J. R., Ramírez M., H., \& Guerra C., V. (2014). Sistema de cubicación para árboles individuales de Tectona grandis L. f. mediante funciones compatibles de ahusamiento-volumen. Revista Mexicana de Ciencias Forestales, 5(21), 58-74.

Tapia, J., \& Návar, J. (2011). Ajuste de modelos de volumen y funciones de ahusamiento para Pinus pseudostrobus Lindl. en bosques de pino de la Sierra Madre Oriental de Nuevo León, México. Foresta Veracruzana, 13(2), 19-28.

Torres R., J. M., \& Magaña T., O. S. (2001). Evaluación de plantaciones forestales. México, D. F.: Limusa.

Trincado, G., \& Burkhart, H. E. (2006). A generalized approach for modeling and localizing stem profile curves. Forest Science, 52(6), 670-682. doi: 10.1093/ forestscience/52.6.670 
Fernández T., E., Fassola, H. E., \& García C., M. (2011). Ecuaciones de volumen total para Populus deltoides de plantaciones del Bajo Delta del Paraná. Revista de Investigaciones Agropecuaria, 37, 172-179.

Uranga-Valencia, L. P., De los Santos P., H. M., Valdez-Lazalde, J. R., López-Upton, J., \& Navarro-Garza, H. (2015). Volumen total y ahusamiento para Pinus patula Schiede ex Schltdl. et Cham. en tres condiciones de bosque. Agrociencia, 49, 787-801.

Zimmerman, D., \& Nunez-Anton, V. (2001). Parametric modelling of growth curve data: An overview. Test, 10(1), 1-73. doi: $10.1007 / \mathrm{BF} 02595823$

Manuscrito recibido el 10 de octubre de 2016

Aceptado el 22 de mayo de 2018

Publicado el 26 de noviembre de 2018
Este documento se debe citar como:

Hernández R., J., Hernández R., A., García C., J., Martínez A., L., Tamarit U., J. C., \& García E., G. G. (2018). Sistema compatible de ahusamiento-volumen comercial para Swietenia macrophylla King (caoba) en Quintana Roo, México. Madera y Bosques, 24(3), e2431441. doi: 10.21829/myb.2018.2431441

Madera y Bosques por Instituto de Ecología, A.C se distribuye bajo una Licencia Creative Commons Atribución-NoComercialCompartirlgual 4.0 Internacional. 